\title{
Interpretaciones infantiles de portadores numéricos en preescolar
}

\author{
Children's interpretations on numerical carriers in preschool Summary
}

\section{Diana Violeta Solares Pineda \\ violetasolares@gmail.com \\ Facultad de Psicología de la Universidad Autónoma de Querétaro, México \\ Claudia Andrea Broitman \\ claubroi@gmail.com \\ Facultad de Humanidades y Ciencias de la Educación, Universidad Nacional de La Plata, Argentina}

\section{Edith Pedroza Velez}

epedrozavz@gmail.com

Facultad de Psicología. Universidad Autónoma de

Querétaro, México

\section{Recepción: 08 Febrero 2020}

Aprobación: 29 Agosto 2020

Publicación: 01 Septiembre 2020

Cita sugerida: Solares Pineda, D. V., Broitman, C. A. y Pedroza Velez, E. (2020). Interpretaciones infantiles de portadores numéricos en preescolar. Archivos de Ciencias de la Educación, 14(17), e082. https://doi.org/10.24215/23468866e082
Resumen: En nuestro estudio analizamos el proceso que siguen niños de preescolar al interpretar portadores de información numérica de uso social. Mediante entrevistas semiestructuradas a diez alumnos estudiamos sus ideas sobre funciones y contenidos de portadores numéricos y sus interpretaciones sobre las informaciones que ofrecen los números presentes. En este trabajo presentamos los resultados en torno a un cartel publicitario.

Las respuestas de los niños reflejan conocimientos y experiencias con actividades sociales que involucran a los portadores, como se observa en las justificaciones a sus interpretaciones. Identificamos particularmente dos recursos que se relacionan y enriquecen mutuamente y en los cuales los niños se apoyaron: las prácticas sociales y las marcas gráficas que acompañan cada número.

Palabras clave: Educación matemática, Preescolar, Portadores numéricos.

Abstract: In our study we analyzed the process followed by preschool children when interpreting social use numerical information carriers.

Through semi-structured interviews conducted on ten students we studied their ideas on functions and numerical carriers' contents, as well as their interpretations about the information provided by the numbers therein. In this study we present the results in connection with an ad.

The students' responses show knowledge and experience around social activities that involve the carriers. The justifications provided to their interpretations about the use of numbers reveal their knowledge and experiences with those usages. We particularly identified two inter related resources leveraged by the children in their interpretations: the social practices and the graphic marks that support each number.

Keywords: Mathematics education, Preschool, Numerical carriers. 


\section{INTRODUCCIÓN}

Esta investigación busca aproximarse a las ideas de los niños respecto a usos de los números en portadores numéricos e identificar el rol de las actividades sociales en sus interpretaciones.

Los números, además de constituir un objeto matemático, son un objeto cultural con el cual los niños interactúan antes de la etapa escolar. La construcción de ideas sobre los usos sociales de los números es producto de su actividad intelectual al participar en situaciones que involucran números en contextos sociales.

Nos preguntamos en qué recursos se apoyan para aproximarse a los usos sociales de los números y cómo interpretan portadores de su entorno. El objetivo es tener un mayor conocimiento sobre las ideas infantiles respecto a los usos de los números presentes en portadores numéricos, para aportar al diseño de situaciones didácticas que recuperen los conocimientos de los niños sobre los usos de los números en distintas actividades de su contexto social.

\section{ANTECEDENTES Y MARCO TEÓRICO}

Los marcos teóricos y antecedentes de esta investigación consideran dos vertientes: los procesos de construcción de los niños sobre los números, y el papel de las interacciones y actividades sociales en la interpretación de textos.

Con respecto a la primera vertiente, investigaciones psicológicas y didácticas de los últimos 30 años han permitido identificar los procesos constructivos de los niños pequeños. Chamorro (2005) señala que la complejidad de los aprendizajes numéricos se debe a la multiplicidad de características de los números, la variedad de tareas que se puede hacer con ellos (clasificar, seriar, contar, transformar, etc.) y sus diferentes significados (cardinal, ordinal, operador, razón, etc.). Ramírez y Block (2006) señalan aspectos que los niños aprenden de la numeración: la representación gráfica, las propiedades sintácticas del sistema de numeración, los significados de los números en el contexto cultural, las propiedades matemáticas involucradas y la naturaleza abstracta de la misma noción.

Diversas autoras han estudiado las ideas infantiles en torno a la producción e interpretación de números escritos, las hipótesis que construyen y los errores constructivos que producen (Lerner, Sadovsky y Wolman, 1994; Alvarado y Ferreiro, 2000; Brizuela, 2000; Alvarado, 2002; Brizuela y Cayton, 2008). Los niños aprenden sobre un sistema de numeración que los precede y sobre el que reflexionan desde sus primeras interacciones (Terigi y Wolman, 2007).

Scheuer et al (2013) exploran los universos numéricos de los niños de primero de primaria al enfrentarlos a problemas en los planos oral y notacional, involucrando cantidades de tamaños distintos y contextos variados. Documentan la diversidad de conocimientos numéricos en niños de la misma edad y en el mismo niño. Algunos estudios focalizan los conocimientos relacionados a los contextos de uso, como los entornos o prácticas donde se usan los números (Sinclair y Sinclair, 1984) o los ambientes y situaciones cotidianas donde se emplean socialmente (Zacañino, 2011). Todas las autoras coinciden en que las experiencias de los niños desde temprana edad les permiten desarrollar ideas respecto al tipo de información que los números proporcionan.

Sinclair y Sinclair (op. cit.) documentan cómo el contexto guía las interpretaciones que los niños hacen de los números e identifican varias de sus funciones. Aunque algunos autores hablan de funciones y otros de usos de los números ${ }^{1}$, utilizamos el término "usos" y distinguimos los siguientes:

- Cardinal: indica la cantidad total de objetos en una colección.

- Código: identifica a un objeto, lugar o persona. 
- Operador: para prever el resultado de una transformación de la cardinalidad operando sobre los números.

- Ordinal: indica el lugar de un objeto en una colección ordenada.

- Medida: expresa la cantidad de unidades de una magnitud continua.

Ferreiro (1982) señala que la escritura tiene presencia en objetos y en relaciones sociales en torno a ellos. Los objetos físicos de uso social que "portan" lo escrito y constituyen el soporte físico de la escritura se denominan "portadores de texto". Quaranta (2003) y Broitman y Kuperman (2004) definen al "portador numérico", término que usaremos, como cualquier objeto cultural que presente números ordenadamente con fines sociales determinados, que pueda funcionar como fuente de información sobre aspectos específicos de los números y del sistema de numeración. Por su parte, Solares (2012) analiza la interacción de los trabajadores agrícolas (incluyendo niños) con diversos "documentos con información numérica".

Aunque un mismo portador puede contener distintos tipos de información, en este estudio enfatizamos la numérica y analizamos cómo el resto (gráfica y textual) aporta elementos para interpretarla. La coexistencia de textos y números en los portadores resultó un desafío para el análisis de las interpretaciones. Por ello, acudimos a estudios que analizan cómo se organiza la información que contienen y cómo se incluyen en actividades sociales.

La perspectiva denominada New Literacy Studies aborda prácticas situadas de lectura y escritura en diversos contextos (Street, 1984; Collins y Blot, 2003): la casa, el trabajo, la escuela, etc. (Baynham, 1995).

Según Barton y Hamilton (1998), la unidad de análisis de este tipo de estudios son las literacy practices, formas culturales en que se usa el lenguaje escrito cotidianamente y que involucran valores, actitudes, sentimientos y relaciones sociales. Afirman que esas prácticas son social e históricamente situadas y que consisten en las interacciones entre personas en torno a textos.

Tales estudios abrieron un debate sobre el supuesto carácter instrumental de la lectura, la escritura y prácticas matemáticas (Kalman y Street, 2009), y son un punto de partida para analizar interpretaciones infantiles sobre números escritos en portadores cotidianos.

\section{Metodología}

Realizamos un estudio cualitativo para indagar ideas infantiles respecto a los usos numéricos en un contexto social. La población estuvo integrada por 10 alumnos de 5 años (seis niños y cuatro niñas) de un grupo de tercero de preescolar de una escuela privada de Querétaro. La escuela informó que esos alumnos podían distinguir números y letras.

Se hicieron entrevistas semiestructuradas con intervenciones propias del método clínico piagetiano. Se buscaba identificar los conocimientos y las experiencias de los alumnos sobre los portadores presentados y los significados que atribuían a informaciones numéricas señaladas. Las entrevistas se realizaron en parejas ${ }^{2}$ y fueron videograbadas. La realización de las entrevistas por parejas obedeció a dos aspectos: por una parte, en congruencia con la perspectiva Literacy Practices, las interacciones de los alumnos en torno al cartel podrían dar cuenta de las formas culturales en que se usa el lenguaje escrito en un momento social e histórico determinado (Barton y Hamilton, 1998); por otra parte, en un pilotaje previo se observó que la interacción entre los participantes permitía la elaboración de respuestas más completas, en contraste con entrevistas individuales, pues cuando los alumnos se encontraban en parejas planteaban sus argumentos, defendían sus ideas o las reformulaban cuando el compañero no entendía o estaba en desacuerdo. Las preguntas que se les plantearon fueron las siguientes:

1. ¿Habías visto antes uno como éste? (mostrando el portador)

2. ¿Dónde lo has visto? / ¿Dónde lo usan? 
3. ¿Para qué sirve? ¿Para qué pusieron ahí los números?

4. ¿Cómo supiste eso?

5. ¿En qué te fijaste?

6. Un niño me dijo que esto era el precio, ¿tú qué piensas? (señalando un número que no expresa el precio).

Los portadores que elegimos fueron un ticket de compra, una bolsa de chocolates y el cartel de un partido de futbol; su presencia en el contexto de los niños se constató previamente en un pilotaje. Aunque en nuestra investigación analizamos los aspectos contextuales, discursivos, gramaticales y léxicos del portador, de cada uno de los números y sus funciones, de las posibles respuestas de los niños y de las posibles intervenciones del entrevistador, en este trabajo presentaremos únicamente los resultados del cartel del partido de fútbol (Figura 1).

FIGURA 1

Cartel del partido de futbol

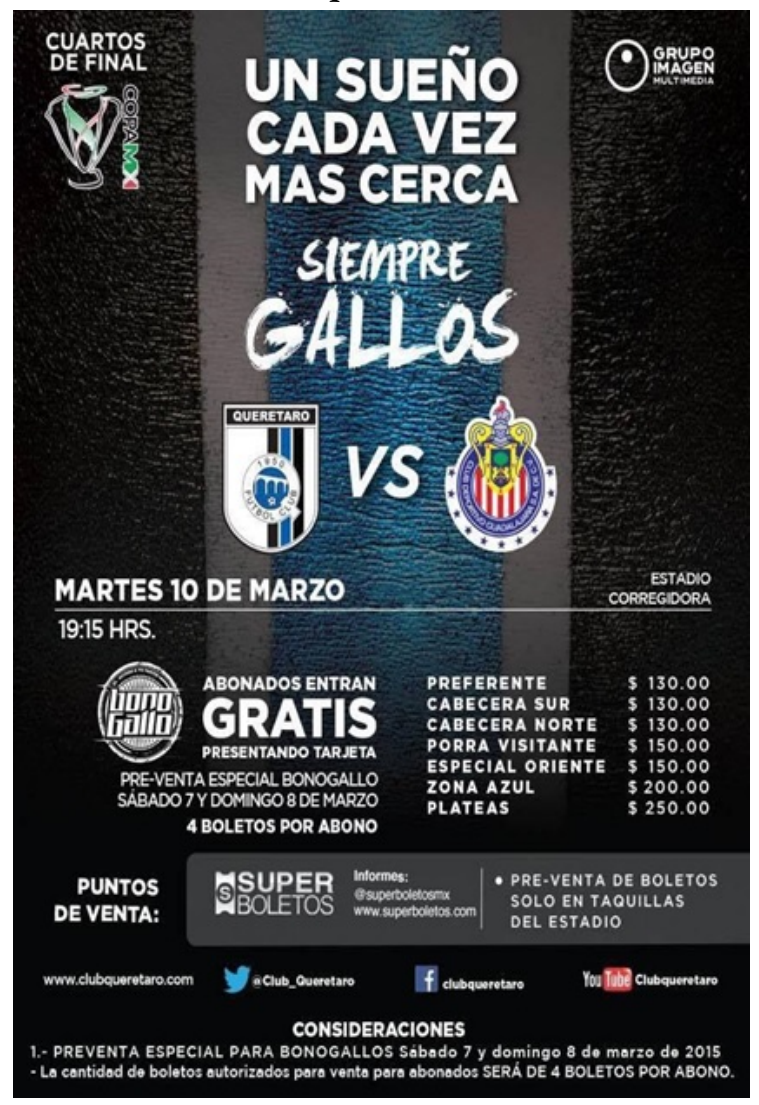

\section{Resultados}

\section{Conocimientos de los niños respecto a la naturaleza cultural del portador}

El procedimiento para explorar la familiaridad de los niños con el portador consistió en presentarles los tres portadores mencionados y preguntarles si habían visto antes alguno similar.

Al preguntarles “¿Dónde lo has visto?” obtuvimos los siguientes tipos de respuesta:

a) 7/10 dijeron haberlo visto en los partidos o en el estadio. 
$\mathrm{E}^{3} \quad$ ¿Y éste que está por acá? (señala el cartel de futbol)

ALFREDO Se pone para los partidos.

E ¿Para los partidos? Oye, ¿y cómo supiste eso?

ALFREDO Porque sé que éstas (señala el escudo del equipo local) son de los Gallos y ése no sé cuál es (se refiere al escudo del otro equipo)

b) 1/10 señaló que había visto uno semejante en su casa.

EMILIO Es un póster.

E ¿Es un póster?

EMLIO Póster son esos adornos que van... (toma el cartel y lo coloca en la pared) y son como unos anuncitos que van en la pared de tu casa.

c) 2/10 dijeron haberlo visto en la escuela.

MARIBEL Ah, y de éstos (señala el cartel) los ponen a veces en la escuela.

Al preguntar “¿Para qué sirve?” respondieron lo siguiente:

a) 5/10 dijeron que era para saber que había un partido o quién jugaba.

MATIAS Sirve esto...

DAVID (lo interrumpe) Para saber...

MATÍAS (Interrumpe nuevamente) Para saber que hoy hay futbol.

E David, ¿para qué sirve esto?

DAVID Para saber qué... quién va a jugar de equipo como Gallos o Chivas o... o América y por eso sirven esos, para que vayan por sus boletos.

b) $2 / 10$ señalaron que era un anuncio sin especificar de qué tipo.

E Oigan, ¿y para qué sirve éste? (señala el cartel)

\section{MARIBEL Para anunciar cosas.}

c) 1/10 sabía que era sobre futbol pero no para qué servía.

E ¿Tú qué crees Roberto?

ROBERTO No sé, es que yo no sé sobre esto (dice en voz baja)

E Sí sabes, tú me dijiste que era de los Gallos.

$[\ldots]$

ROBERTO Y también en los estadios.

E ¿En los estadios? ¿Por qué?

ROBERTO grandototes. Porque le ponen de éstos (señala el cartel) pero nada más que

d) $2 / 10$ no respondieron.

Las respuestas corroboraron que conocían al portador. Los conocimientos en los que se apoyan podrían estar vinculados a tres aspectos: las actividades que tienen lugar en torno al portador, sus características (tamaño, tipo de papel, distribución de la información) y las pistas gráficas que permiten interpretar la información escrita. 
Presenciar un partido y comprar boletos fueron actividades que señalaron los entrevistados. Al mencionarlas manifestaron sus conocimientos, como en las interacciones de las parejas 1 y 5 que se presentan a continuación.

En el caso de la pareja 1 la participante refiere que el partido ya tuvo lugar, y comenta que el desempeño del equipo local es muy bueno, lo que provoca la reacción de su compañero.

Pareja 1.

ROBERTO Mmm... ¡wow! ¿van a jugar los Gallos contra Chivas? (señala los escudos de los equipos de futbol mientras dice su nombre). No,

MARIBEL $[\ldots]$ ese ya... ya pasó, fue ayer.

MARIBEL ¡Gallos Blancos! Siempre están en los partidos y siempre ganan.

ROBERTO No es cierto.

MARIBEL Y es verdad.

ROBERTO Contra... contra León no ganaron, perdieron.

En la pareja 5 David describe el propósito del cartel: saber quiénes son los equipos que se enfrentan y así poder ver el partido. Aunque David detalla cuáles son las modalidades para ver el encuentro, ambos participantes coinciden al señalar la importancia de la compra de boletos para asistir al estadio.

Pareja 5.

E David, ¿para qué sirve esto?

DAVID Para saber qué... quién va a jugar de equipo como Gallos o Chivas o... o América y por eso sirven esos, para que vayan por sus boletos.

E ¡Wow!

MATÍAS Sí, van... sí, toman sus boletos para entrar al futbol.

$[\ldots]$

DAVID Es que... es que algunos señores ponen unos pegados (señala el cartel) unos papelitos para que vean quién se enfrentan y para que nosotros los veamos en vivo o en la televisión, para que lo sepamos, para comprarle los boletos.

En resumen, los indicios del cartel que permiten a los niños interpretar la información que contiene son imágenes, símbolos y palabras específicas. Incluso sin la imagen de un balón o la palabra "futbol", los participantes reconocieron que el anuncio se relaciona con ese deporte. Las respuestas constatan la utilidad de los escudos, los cuales fueron reconocidos y permitieron vincular al portador con un conjunto de actividades sociales; en este caso, con la compra de boletos a un partido y la asistencia o visualización de este.

La familiaridad de los niños con este tipo de portadores les hizo identificar la función del cartel. Mencionaron la palabra "anuncio" y la vincularon con informar sobre un evento. Esta referencia podría relacionarse con la presencia de publicidad en revistas, periódicos o anuncios espectaculares en su contexto. Los niños interactúan con publicidad de tales actividades y pueden elaborar ideas respecto a lo que comunican este tipo de portadores.

\section{Recursos en los que se apoyan los niños para interpretar la información numérica}

Identificamos tres aspectos que aparecieron como referencia y/o como justificación de la interpretación que hicieron de los datos numéricos: 1) aspectos contextuales (los conocimientos que tienen sobre el futbol y las actividades sociales que le rodean); 2) discursivos (las informaciones o pistas gráficas que contiene el portador); 3) gramaticales y léxicos (la lectura de algunas palabras incluidas en el portador). 
Para efectos del análisis diferenciamos esos tres aspectos, aunque se presentan de manera conjunta cuando los niños interpretan la información numérica.

\section{Aspectos contextuales: actividades sociales y conocimientos del futbol}

Los diez participantes recurrieron a experiencias previas al cartel, como haber visto o jugado futbol. Aunque podrían apoyarse en sus conocimientos sobre carteles en general, lo que los ayudó a plantear sus conjeturas fue conocer "lo que debería decir el cartel acerca del partido".

Un ejemplo de esto es el intercambio de la pareja 5, quienes recurren a su experiencia comprando boletos para interpretar la lista de precios de los boletos.

Ambos relacionaron la lista de números con la venta de boletos. David describe el procedimiento que se sigue para comprarlos y señala el tipo de información en el boleto adquirido, mientras Matías refiere que la lista de precios permite ver el costo de la entrada al partido.

\section{Aspectos discursivos: pistas gráficas en el portador}

Entre las pistas gráficas del portador consideramos los escudos, los logotipos y los signos que permiten interpretar la información numérica. Estas pistas fueron señaladas por los diez participantes al menos una vez a lo largo de las entrevistas, y les permitieron argumentar sus planteamientos, como se muestra con Emilio, quien se apoyó en el símbolo de pesos para justificar su respuesta.

E Quiero que se fijen en estos números que están aquí, (señala los precios de los boletos) ¿Por qué los pusieron ahí?

EMILIO Creo que es la puntuación del póster.

E ¿La puntuación de qué?

EMILIO O sea, es... creo que es el póster de un partido porque puede ser de un partido. Entonces aquí viene cuántos goles anotaron y eso.

E ¿En serio? Oye Felicia, ¿tú crees que aquí...

EMILIO O la puntuación.

E ¿Los puntos o los goles que me dijiste?

EMILIO Puntos... puntos también hay otras cosas que te dan puntos.

E Ok. Muy bien. Felicia, ¿por qué crees que pusieron...?

EMILIO (interrumpiendo) ¡Ah, ya sé! Cuánto costó el partido.

E ¿Cómo supiste?

FELICIA Ah... es cuántos goles metieron. (Señala las columnas con ceros en los precios)

EMILIO No es cierto.

FELICIA Yo ya le atiné.

EMILIO No es cierto.

FELICIA Cuántos goles metieron.

EMILIO No, ¿te digo por qué? Porque el... no pueden meter así... ¡Esto es un número trescientos! (señala el 130) ¡No pudieron meter trescientos! ¡Imagínate trescientos goles!

E Pero Felicia dice que estos son los goles que metieron (señala las columnas de ceros) no éste de acá (señala las columnas de números escritos antes del punto)

EMILIO Pero yo digo que esto es lo que costó el partido porque mira aquí hay un numerito, estas cosas se llaman insignios de... de... de lo que cuestan. (Señala símbolo de pesos) 
Emilio fue modificando sus interpretaciones. En un principio señaló que los números referían la puntuación del partido o los goles. Finalmente indica que se trata del costo del boleto, advirtiendo la presencia del "insignio de lo que cuestan" (símbolo de pesos: \$). Además de la pista gráfica, su conocimiento sobre el futbol le permitió rechazar que los números indican la cantidad de goles.

\section{Aspectos gramaticales y léxicos: lectura de palabras}

Este recurso fue empleado por cuatro de los diez participantes. Los niños recurrían a la lectura de palabras cuando trataban de interpretar la información numérica, particularmente se usó para las fechas, pues los números correspondientes al número de día y de año se encontraban escritos entre un conjunto de palabras. Para estos niños la lectura del mes o el día resultaba un indicio que les permitía elaborar sus ideas.

E Oigan, ¿y ya se fijaron que acá hay otro numerito? (señala la fecha del partido: 10 DE MARZO)

MARIBEL Ah... diez.

E ¿Ése para qué está ahí?

MARIBEL Mar...tes diez de marzo. ¡Martes diez de mayo!

ROBERTO ¡De marzo!

MARIBEL De marzo.

$[\ldots]$

E Roberto, ¿Para qué pusieron ese número ahí? (señala el 10 en la fecha del partido)

ROBERTO Mmmm.. de... para...dice ma.... artes... diez... de mar... zo. Martes diez de marzo.

MARIBEL Eso yo ya lo leí.

E ¿Y para qué lo pusieron?

MARIBEL Para ver qué día es.

ROBERTO Para saber cuándo es el partido Gallos contra Chivas.

Nuestra hipótesis es que la lectura del día y el mes constituye un recurso viable para la interpretación de las fechas, pues éstas son escritas constantemente por los alumnos en el salón de clases.

En síntesis, podemos apreciar que los niños no hicieron uso exclusivo de un solo recurso, sino que hay una interrelación entre dos o incluso los tres aspectos. En el ejemplo de Emilio y Felicia se observa cómo los conocimientos en torno al futbol y los carteles permitieron a Emilio elaborar una primera interpretación sobre la lista de precios: "son los goles". Esta interpretación fue para Felicia el punto de referencia para su interpretación: como sabe que el resultado de un partido puede quedar en cero goles, identificó los ceros que siguen al punto decimal del precio como "cuántos goles metieron". Sin embargo, Emilio advierte el símbolo de pesos y reelabora: la lista indica lo que costó la entrada al partido.

La interacción entre Emilio y Felicia nos permite identificar que, aunque el indicio gráfico fue sumamente importante para defender la idea de que la lista hacía referencia al costo de la entrada, esta idea fue precedida por otras que estaban apoyadas en el conocimiento de las actividades sociales implicadas en el futbol.

\section{Interpretaciones de los niños en torno a la información numérica}

Sobre el cartel del partido de futbol seleccionamos cuatro informaciones numéricas: dos fechas en formatos distintos, los precios de los boletos y la hora del partido. Esas informaciones hacen referencia al uso de los números como medida y como ordinal. Organizamos las respuestas en tres apartados de acuerdo 
con el tipo de interpretación: de las fechas, de la hora y de los precios. En cada uno se presentan categorizadas las respuestas a las preguntas “¿Para qué pusieron este número?” y “¿Cómo supiste o en qué te fijaste?".

\section{Fechas}

El cartel presentaba dos fechas: la del partido (Figura 2) y la de la preventa de boletos (Figura 3). Las respuestas que dieron los niños a esos datos nos hacen suponer que les es más fácil reconocer la fecha cuando la escritura incluye al año: cinco de los diez niños identifican una fecha en "martes 10 de marzo", mientras que siete identificaron una en "sábado 7 y domingo 8 de marzo de 2015 "

FIGURA 2

\section{MARTES 10 DE MARZO}

Detalle del cartel: fecha del partido

FIGURA 3

\section{Sábado 7 y domingo 8 de marzo de 2015}

Detalle del cartel: fecha preventa de boletos

Al preguntarles “¿Para qué habrán puesto estos números?” señalando la fecha de preventa de boletos, obtuvimos los siguientes tipos de respuesta: a) es la fecha, b) es el costo del boleto, c) es la hora, d) no respondió.

a) $7 / 10$ comentaron que esta escritura hacía referencia a la fecha.

E Oigan... y aquí ustedes me dijeron que estaba el dos mil quince. Pero ya se fijaron que hay otros números acá (señala el renglón donde está escrita la fecha de la preventa), ¿para qué son todos esos números?

ROBERTO [...]

MARIBEL Sábado siete y... do...mingo...

ROBERTO Ah, es para sábado siete.

MARIBEL ¡Siete!

ROBERTO Es que aquí...

MARIBEL El siete y domingo.

Es... es el sábado... fue sábado siete y domingo ocho... marzo do... dos mil quince.

E ¿Y eso para qué es?

MARIBEL Para saber en qué fecha va a ser, qué tal si fue en el dos mil catorce $\mathrm{y} . .$. qué tal que se va en el dos mil quince.

b) 1/10 mencionó que los números indicaban el costo del boleto.

E (Señala los números en la fecha para venta de boletos: 7, 8 y 2015). Aquí dice uno, aquí dice otro y acá otro, ¿para qué los pusieron ahí?

DAVID Para saber cuánto costaba su dinero.

MATÍAS ¡Los boletos!

c) 1/10 sugirió que era la hora a la que debían llegar los jugadores y el entrenador al partido. 
MATÍAS No.

¿Puede decir cuánto cuesta entonces?

DAVID No. [...] Mmmm... para los siete (se refiere al número 7 en la fecha de preventa) en punto van a llegar su entrenador y los otros, y luego van a ver su boleto para que los... para que los alumnos a los... los papis y los hijos lleguen muy temprano y ellos van a... van a estar ahí a... como a las diez (señala el 10 en la fecha del partido "10 DE MARZO") van a estar.

E Y luego, ¿éste de acá para que lo pusieron? (señala el 2015)

DAVID Ya no me acuerdo.

d) $1 / 10$ no respondió.

Después de que los niños interpretaron para qué servían los números de la fecha, se les preguntó “¿Cómo supiste?” o “¿En qué te fijaste?”, para saber en qué se apoyaban para hacer sus interpretaciones.

Para la fecha del partido cinco de los diez alumnos justificaron sus interpretaciones, mientras que para la fecha de preventa fueron nueve. En el cuadro 1 se reportan ejemplos de las justificaciones para cada una de las fechas. La diferencia en la cantidad de respuestas acertadas nos permite corroborar que las diferencias en la escritura de las fechas inciden en la manera en que los niños las interpretan.

\section{CUADRO 1}

Justificaciones a la interpretación acertada de las fechas

\begin{tabular}{|c|c|}
\hline Martes 10 de marzo & Sábado 7 y domingo 8 de marzo de 2015 \\
\hline $\begin{array}{l}\text { a. Reconocen la escritura } \\
\text { de la fecha (4 niños) } \\
\text { MARIBEL: (...) es que como aquí } \\
\text { decía "martes diez de mayo" (repasa } \\
\text { con su dedo el renglón donde está } \\
\text { escrita la fecha) entonces, entonces } \\
\text { pues debe de ser ese día. } \\
\text { E: ¿Por qué? } \\
\text { MARIBEL: Porque hay muchos días } \\
\text { que pueden ser los partidos. Por } \\
\text { ejemplo ayer en la noche hubo un } \\
\text { partido. }\end{array}$ & $\begin{array}{l}\text { a) Está escrito el año (4 niños) } \\
\text { EMILIO: Este es el número de año } \\
\text { porque dice dos mil catorce (señala } \\
\text { el 2015)... es el día del partido de } \\
\text { Gallos Blancos contra Pachuca. } \\
\text { b) Se parece a la fecha del salón de } \\
\text { clase (3 niños) } \\
\text { EMMA: Es la fecha. } \\
\text { ALFREDO: La fecha. } \\
\text { E: ¿La fecha? Oh, ¿y cómo supiste } \\
\text { eso Emma? } \\
\text { EMMA: Porque en mi salón está lo } \\
\text { mismo. (Mientras contesta mira por } \\
\text { la ventana en dirección a su salón } \\
\text { de clase) }\end{array}$ \\
\hline
\end{tabular}

Para los niños es más fácil reconocer la fecha cuando en su escritura se incluye el año. Probablemente al permanecer fijo durante un periodo de tiempo mayor, el año constituya un indicio más fuerte para reconocer la escritura de la fecha. 


\section{Hora del partido}

FIGURA 4

Detalle del cartel: hora del partido

\section{9:15 HRS.}

Cuando preguntamos a los niños “¿Para qué habrán puesto los números 19:15?” obtuvimos los siguientes tipos de respuesta: a) es la hora, b) es el precio, c) es el día, d) es para numerar boletos, e) no respondió.

a) 4/10 participantes dijeron que era la hora.

E Estos números que están acá (señalando la hora del partido) Estos números de aquí para qué sirven.

MARIBEL Ah... sería para...

ROBERTO Para la hora.

b) 3/10 dijeron que era el precio.

E Un niño me dijo que aquí (señala la hora) dice el precio.

ALFREDO ¿El precio de qué?

E (Asintiendo con la cabeza) El precio.

ALFREDO El precio de los boletos.

E Ajá, me dijo aquí dice el precio ¿ustedes qué creen?

ALFREDO Que sí.

Los niños aceptaron la interpretación provista por la entrevistadora en una intervención dirigida a la contra-argumentación, la cual se realizó porque permanecieron en silencio luego de que la entrevistadora planteara la pregunta varias veces. En lugar de oponerse, aceptaron su interpretación, lo cual podría significar que ellos creen que se trata de precios o que tienen otra idea pero no se atrevieron a discutir la voz del adulto.

c) $1 / 10$ señaló que indicaba el día.

E Oigan... ¿y este (señala la hora del partido) para qué lo pusieron?

EMILIO Ah... ése es el día

E ¿Tú qué opinas Felicia?

FELICIA Que sí.

d) 1/10 mencionó que servía para numerar los boletos.

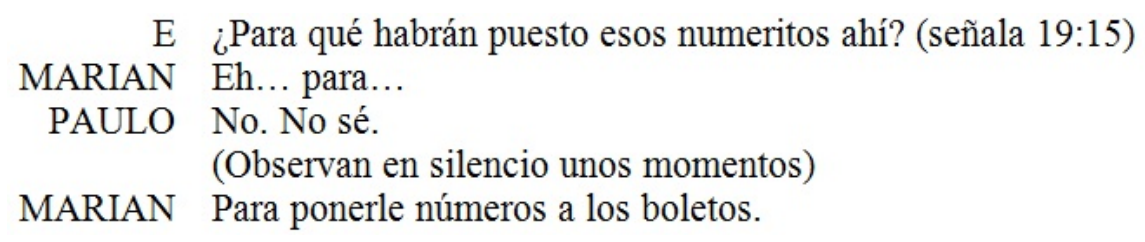

e) $1 / 10$ no respondió.

Al preguntar a los niños “¿Cómo supiste?” o “¿En qué te fijaste?” obtuvimos las siguientes respuestas: a) por los dos puntos, b) adivinamos, c) no respondió.

a) 5/10 respondieron que habían advertido los dos puntos. 
EMILIO Día, hora y minuto. Ah no, es... minuto, hora... minuto, hora, segundo y... y ya.

E ¿Y cómo supiste que esto era la hora? Qué tal que nos...

EMILIO Porque estos puntitos, estos puntitos son los segundos... éstos son los minutos (señala el 19), segundos (señalando los dos puntos) y ésta es la hora (señala el 15). [...] Cuando pasan sesenta de estos, sesenta... cuando parpadea sesenta veces esto (los dos puntos) cambia de hora.

b) $1 / 10$ respondió que había adivinado.

\section{E ¿Sí es para la hora? ¿Por qué Maribel? \\ MARIBEL No sé. \\ ROBERTO Yo quién sabe, sólo lo adiviné.}

c) $4 / 10$ no respondieron.

Entre los participantes que no proporcionaron justificación, Alfredo y Emma aceptaron la idea planteada por la entrevistadora respecto a que los números de la hora indicaban precios. Aunque ellos no señalaron los dos puntos, sí interpretaron de manera separada las cantidades. Leyeron el 19 como sesenta y el 15 como cincuenta y mencionaron que esos podrían ser los precios de los boletos. Cabe apuntar que "sesenta" y "cincuenta" son cantidades factibles de representar un precio, no así una hora, por lo que es muy probable que estos dos alumnos efectivamente consideren que esos números representen un precio.

Felicia apoyó la idea planteada por su compañero, pero no expresó una justificación sobre por qué creía que los números hacían referencia al día del partido.

Matías argumentó la imposibilidad de que en 19:15 dijera la hora, pero no planteó una idea propia.

Esta información numérica resultó más difícil de interpretar por parte de los niños. El número de respuestas acertadas en la interpretación del uso de los números fue el más bajo. Nuestra conjetura respecto a que los dos puntos serían un apoyo para que los niños hicieran su interpretación no fue acertada. Sólo dos de los cinco niños que advirtieron esta marca gráfica la vincularon con la escritura de la hora. No obstante, la interpretación que hacen de los dos puntos resulta interesante, pues le atribuyen una función como separador: cuando están escritos los dos puntos se separa la información.

Solamente preguntamos por este uso numérico en el cartel del partido; su presencia en los otros portadores no fue abordada en la entrevista al considerarse una información mucho más compleja.

\section{Lista deprecios}

FIGURA 5

Detalle del cartel: precios de los boletos

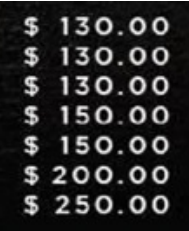

Los números que indican precios fueron más identificables por los niños que el resto de la información numérica. Obtuvimos los siguientes tipos de respuesta: a) es el costo o el pago, b) es el marcador, c) es para calificar, d) no respondió.

a) 7/10 señalan que era el costo o cuánto tenían que pagar por el boleto. 
E Oigan... ¿y ustedes por qué creen que pusieron estos números aquí? (señala los precios de los boletos)

MATÍAS Ah... son demasiados

[...]

E ¿Y entonces estos números los pusieron para qué Matías?

MATÍAS Sí, para ver cuánto cuesta la entrada del... del partido.

b) 2/10 mencionan que los números refieren el marcador del partido.

E Pero acá (señala la lista de precios) cómo supiste que ahí dice cuántos goles metieron.

FELICIA Porque aquí tiene números, cuántos goles metieron.

E A ver enséñame.

FELICIA Mira aquí está un 3 (en el precio \$130.00), porque ahí tiene que estar.

c) $1 / 10$ afirma que el número es para calificar.

E ¿Tú qué crees Alfredo? Ésos números que nos dice Emma dicen que es para empatar.

ALFREDO Mmmm... pues... para calificar.

$[\ldots]$

E ¿Por qué Alfredo?

ALFREDO $^{4}$ Porque así... aquí... sacan los números que anotaron los jueces 0 no sé si el juez.

Preguntamos a los niños en qué se fijaron al interpretar la escritura de los precios y obtuvimos los siguientes tipos de respuesta: a) el símbolo de pesos, b) los ceros, c) me dijeron, d) no respondió.

a) 5/10 señalaron el símbolo de pesos como un indicio que les permitió identificar el precio.

ROBERTO Para sāber cuánto cuestan cada partido.

E ¿Y cómo supiste eso?

ROBERTO Porque aquí hay (señala los signos \$) algo de... de como... de dinero.

E Ah... a ver enséñamelo, ¿cuál es?

ROBERTO Este (señala el símbolo \$)

b) 2/10 repararon en las columnas de ceros que se encontraban al final de los precios.

E Fíjense, en éste (se refiere al cartel) pusieron estos números aquí (señala los precios de los boletos) ¿ya los vieron todos los que están aquí? ¿Para qué los pusieron ahí?

EMMA Para empatar.

E ¿Para empatar? A ver, dónde Emma. Señálamelo.

EMMA Aquí (señala los ceros que indican los centavos)

c) $1 / 10$ comentó que lo supo porque le dijeron.

E ¿Y entonces estos números los pusieron para qué Matías?

MATÍAS Sí, para ver cuánto cuesta la entrada del... del partido.

E Órale y cómo supiste eso.

MATÍAS Ah... porque yo un día fui al estadio y, y dijo un señor que eso es cierto. 
d) 2/10 no respondieron.

En síntesis, pudimos ver cómo los niños entrevistados pudieron analizar la información del cartel y dar interpretaciones plausibles relacionadas con el futbol. Aun aquellas interpretaciones erróneas estaban dentro de lo que podría decir el cartel y no hubo ninguna respuesta que no fuera así. La respuesta de Emma es un buen ejemplo de ello, pues es factible que el resultado de un partido quede empatado en cero goles (0-0).

Los niños interpretaron escrituras que incluían signos de puntuación. Varios atribuyeron una función de "separador" al punto o los dos puntos. Estos signos llevaban a los niños a interpretar de manera distinta los números, como si fueran escrituras separadas, lo cual se aprecia en el siguiente registro:

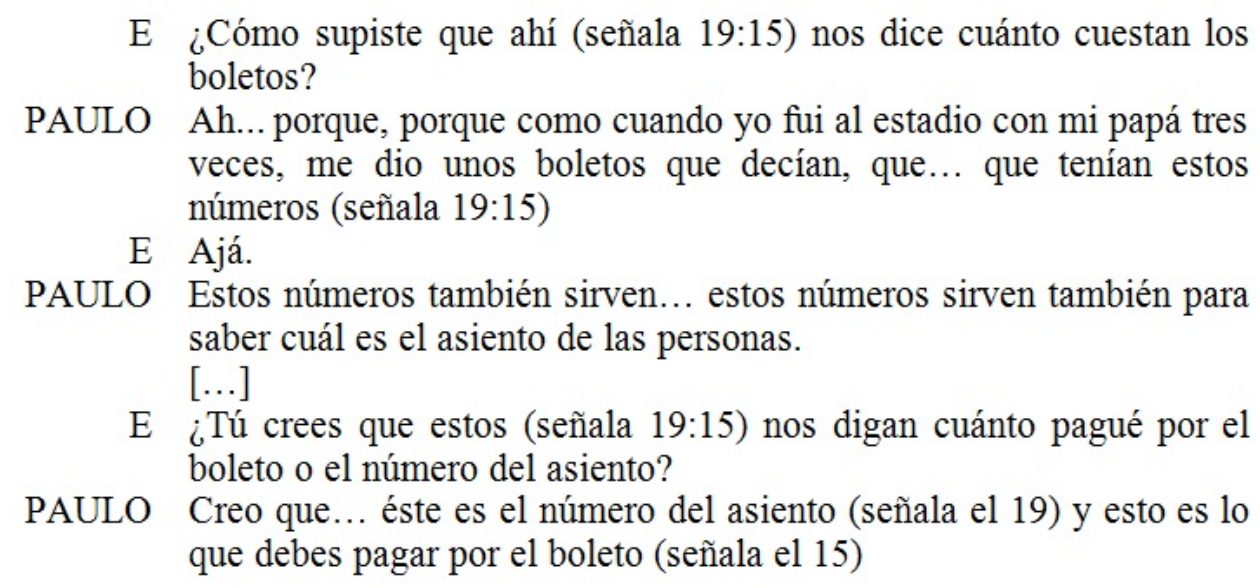

Explicaciones como éstas fueron reportadas por Brizuela y Cayton (2008) en una investigación sobre la manera en que niños preescolares y de primer grado de primaria interpretan signos de puntuación en escrituras numéricas. Señalan que el 52\% de los niños refirieron que el punto se usaba para separar y el 30\% mencionó que el punto hacía que un número fuera distinto. En el caso de nuestro estudio, algunos niños interpretaron que tanto el punto como con los dos puntos servían para separar.

Cabe recordar que nosotras preguntamos en una sola ocasión por una escritura numérica de la hora y que 4/10 participantes la interpretaron de manera correcta. Es decir, aunque fue la que tuvo menor cantidad de aciertos, no podemos decir que sea un tipo de información totalmente desconocida para los niños.

Respecto a los precios, es una escritura con la que tienen contacto de manera frecuente en su entorno social, particularmente en actividades de compra-venta.

\section{Conclusiones}

En nuestro estudio se provoca que los niños interpreten la información numérica escrita en los portadores. Aunque no son los destinatarios del cartel, tienen conocimientos que les permiten realizar un análisis del propósito de la información contenida. Identificamos un rasgo común que permitió a los niños elaborar interpretaciones acerca de la información numérica: las actividades sociales implicadas en el futbol. Los niños manifiestan un bagaje de experiencias vinculadas al futbol y hacen referencia a ellas cuando tratan de interpretar la información numérica del cartel.

Los participantes de nuestro estudio interpretaron el tipo de información apoyándose en tres recursos: sus conocimientos acerca de las actividades en torno al futbol, las marcas gráficas y la lectura de palabras.

Los resultados permiten identificar también marcas gráficas en el cartel que funcionan como indicios que posibilitan la interpretación del significado de los números en el contexto de un cartel sobre un partido de futbol: el símbolo de pesos, los dos puntos y el punto. 
La lectura de palabras permitió a algunos plantear sus ideas respecto al significado de los números en las fechas y justificar sus interpretaciones.

El estudio permite constatar lo que reportaron Sinclair y Sinclair (1984): los niños son sensibles a la variedad de usos que tiene el número; en un mismo portador identifican que los números comunican informaciones distintas.

Los niños pudieron atribuir propósitos diversos vinculados con el contexto a la información presentada en el cartel. El reconocimiento del portador como un anuncio y sus saberes acerca del futbol se conjugaron para interpretar la información de los números en el cartel. En suma, la interpretación de la información numérica y el reconocimiento de la variedad de significados del número es una tarea que los niños enfrentaron apoyándose en conocimientos de tipo social y contextual.

Apropiarse de los números no es solamente atender las características del sistema de numeración, también es advertir la multiplicidad de actividades que permite realizar en el contexto social. Apostamos al diseño de futuras producciones didácticas que vean a los números como objetos sociales. Estas investigaciones requerirán aportes de la didáctica de la matemática y de la de lectura y escritura.

\section{REFERENCIAS}

Alvarado, M. (2002). La construcción del sistema gráfico numérico en los momentos iniciales de la adquisición del sistema gráfico alfabético (Tesis doctoral inédita). CINVESTAV-IPN, Ciudad de México

Alvarado, M. y Ferreiro, E. (2000). El análisis de nombres de números de dos dígitos en niños de 4 y 5 años. Lectura y Vida. Revista Latinoamericana de Lectura, 21(1), 6-17

Barton, D y Hamilton, M. (1998). Local literacies. Reading and writing in one community. Londres: Routledge

Baynham, M. (1995). Literacy practices: Investigating literacy in social contexts. Londres: Longman

Brizuela, B. (2000). Algunas ideas sobre el sistema de numeración escrito en niños pequeños. En N. Elichiry (Ed.), Aprendizaje de niños y maestros. La construcción del sujeto educativo (pp. 15-26). Buenos Aires: Ediciones Manantial

Brizuela, B. y Cayton, G. (2008). The roles of punctuations marks while learning about written numbers. Educational Studies In Mathematics, 68(3), 209-225

Broitman, C y Kuperman, C. (2004). Interpretación de números y exploración de regularidades en la serie numérica. Propuesta didáctica para primer grado: La lotería. Buenos Aires, OPF y Oficina de Publicaciones de la Facultad de Filosofía y Letras de la UBA, Argentina

Collins, J.y Blot, R. (2003). Literacy and literacies: Texts, power, and identity. Nueva York: Cambridge University Press

Chamorro, C. (2005). La construcción del número natural. En C. Chamorro (Coord.), Didáctica de las Matemáticas para la Educación Infantil (pp. 141-180). Madrid: Pearson Education

Ferreiro, E. (1982). Los procesos constructivos de apropiación de la escritura. En E. Ferreiro y M. Gómez (Comps.), Nuevas perspectivas sobre los procesos de lectura y escritura (pp. 128-154). México-Argentina: Siglo XXI Editores

Kalman, J. y Street, B. (2009). Lectura, escritura y matemáticas como prácticas sociales: Diálogos con América Latina. México: Siglo XXI Editores /CREFAL

Lerner, D., Sadovsky, P. y Wolman, S. (1994). El sistema de numeración: un problema didáctico. En C. Parra e I. Saiz (Comps.), Didáctica de Matemáticas (pp. 95-184). Buenos Aires: Paidós

Pedroza, E. (2016). Interpretación de portadores numéricos en alumnos de preescolar. Tesis de maestría. Santiago de Querétaro, Facultad de Psicología, Universidad Autónoma de Querétaro, México

Quaranta, E. (2003). La serie numérica oral. En Orientaciones didácticas para el nivel inicial-2a parte. (pp. 73-98). Buenos Aires: DGCyE-Subsecretaría de Educación

Ramírez, L. y Block, D. (2006). Análisis de situaciones didácticas para el aprendizaje del número en preescolar. Documentos DIE CINVESTAV, 59 
Scheuer, N., Santamaría, F. y Bordoli, C. (2013). Una aproximación al universo numérico de chicos que inician la escolaridad primaria. En Broitman, C. (Comp.), Matemáticas en la escuela primaria I. Números naturales y decimales con niños y adultos (149-171). Buenos Aires: Paidós

Sinclair, A. y Sinclair, H. (1984). Las interpretaciones de los niños preescolares sobre los números escritos. Human Learning, 3, 173-184

Solares, D. (2012) Conocimientos matemáticos de niños y niñas jornaleros agrícolas migrantes. Tesis doctoral. México, Departamento de Investigaciones Educativas, Centro de Estudios Avanzados del Instituto Politécnico Nacional

Street, B. (1984). Literacy in theory and practice. Cambridge: Cambridge University Press

Terigi, F. y Wolman, S. (2007). Sistema de numeración: consideraciones acerca de su enseñanza. Revista Iberoamericana de Educación, 43, 59-83

Zacañino, L. (2011). Las notaciones numéricas en contextos de uso diferentes. (Tesis de maestría). Facultad de Psicología de la Universidad de Buenos Aires, Buenos Aires

\section{Notas}

1 En la tesis de la que surge este trabajo (Pedroza, 2016) se documentan las concepciones en torno a "funciones" y "usos", así como las distintas funciones o usos del número que cada autor considera. Nuestra clasificación surge a partir de este análisis.

2 La docente de la escuela sugirió los grupos buscando que los alumnos tuvieran niveles de conocimiento y expresión oral similares.

3 La letra E se refiere a "Entrevistadora".

4 El participante refiere que son para calificar mencionando a los jueces; creemos que se refiere al método empleado en algunos deportes donde se promedian las puntuaciones otorgadas por varios jueces. 